\title{
The Value of Tyrer-Cuzick Versus Gail Risk Modeling in Predicting Benefit from Screening MRI in Breast Cancer
}

\author{
(D) Anthanasios Sevdalis ${ }^{1}$, (1) Xiaoyan Deng², (1) Dipankar Bandyopadhyay ${ }^{2}$, (10) Kandace P. McGuire ${ }^{1}$ \\ ${ }^{1}$ Division of Surgical Oncology, Department of Surgery, Virginia Commonwealth University, Virginia, USA \\ ${ }^{2}$ Department of Biostatistics, Virginia Commonwealth University, Massey Cancer Center, Virginia, USA
}

\begin{abstract}
Objective: Breast cancer is the most commonly diagnosed malignancy in US women. Risk assessment tools such as the Gail and Tyrer-Cuzick (TC) models calculate risk for breast cancer based on modifiable and non-modifiable factors in order to guide screening and prevention for high-risk patients. Screening with magnetic resonance imaging (MRI) in addition to mammography is recommended in high-risk patients ( $>20 \%$ lifetime risk on TC or other familial based models). Currently, no published data indicate these recommendations improve cancer detection.

Materials and Methods: With the aim to determine what percentage lifetime risk (LR\%) is associated with a statistically significant increase in cancer detection, the Virginia Commonwealth University (VCU) breast imaging database was reviewed to identify patients who received screening MRI.

Results: The receiver operating characteristics (ROC) curves for the Gail and TC models and the rate of cancer detection correlated to $20 \% \mathrm{LR} \%$ were calculated. The Gail model was considered the control model as it is NOT considered a validated screening tool for MRI. TC is not more accurate than Gail when predicting benefit of breast MRI screening. (area under the curve (AUC): $0.6841,0.6543$ respectively, $\mathrm{p}=0.828$ ). Univariate analysis failed to demonstrate a statistically significant relationship between the Gail or TC LR \% and diagnosis of breast cancer when using $20 \%$ as the cutoff for high-risk classification ( $\mathrm{p}=1.0,0.369$ respectively). Neither the TC nor the Gail risk calculators demonstrated a significant correlation between risk and the likelihood of diagnosis of breast cancer when screened with MRI.
\end{abstract}

Conclusion: Larger cohort studies are necessary to determine the risk percentage most predictive of a breast cancer diagnosis using MRI as screening. Keywords: Breast cancer, breast cancer screening, MRI, risk factors

Cite this article as: Sevdalis A, Deng X, Bandyopadhyay D, McGuire KP. The value of Tyrer-Cuzick Versus Gail Risk Modeling in Predicting Benefit from Screening MRI in Breast Cancer. Eur J Breast Health 2022; 18(1): 79-84

\section{Key Points}

- Currently the Tyrer-Cuzick model is used for determination of MRI eligibility for high-risk patients whereas the Gail model guides eligibility for chemoprevention.

- Our study demonstrated that there might not be any additional predictive value using the Tyrer-Cuzick versus Gail model when determining screening MRI breast eligibility.

- The $20 \%$ lifetime risk, as calculated by Tyrer-Cuzick, did not appear to lead to a greater detection of breast cancers over our control, the Gail model. This calls into question the $20 \%$ cutoff but would require larger studies to determine a more appropriate cutoff value.

\section{Introduction}

Breast cancer is the most commonly diagnosed malignancy in women in the United States and the second most common cause of cancer death among women worldwide (1). On average, a woman's risk for developing invasive breast cancer in the United States (US) is approximately 1 in 8 or about $12.5 \%$. This risk increases with age, with a woman aged 70 being almost 10 times more likely to develop breast cancer in the next five years as compared to a woman in her 30s (2). There are several other factors, both modifiable and non-modifiable, that can increase a woman's risk for developing breast cancer. Such modifiable factors include obesity, alcohol consumption, activity level, parity, breastfeeding, radiation therapy and use of hormone replacement therapy (HRT) (3). Non-modifiable factors include genetic mutations, family history of breast cancer, prior history of atypical lesions, as well as race and age $(4,5)$. 
Studies have demonstrated that early detection of breast cancer decreases the morbidity and mortality of the disease (6). Routine screening with mammography has decreased mortality, especially in women aged 50 to 69 years $(7,8)$. In fact, most women with clinically occult disease are diagnosed with breast cancer by mammographic screening alone. While breast cancer screening primarily relies on mammography, there are proven benefits in screening for breast cancer with contrastenhanced breast magnetic resonance imaging (MRI). Contrastenhanced breast MRI has superior sensitivity to mammography (911). Even when adding ultrasound to mammography, the two have relatively lower specificity and sensitivity to mammogram and MRI $(12,13)$. Some factors that have hindered the wider use of MRI for screening for breast cancer are its high cost, need for heavy metal (Gadolinium) contrast, the limited availability of MRI scanners and its low specificity for breast cancer detection. The specificity of MRI in multiple studies remains around $70 \%$. Increased sensitivity and decreased specificity, as compared to mammography, results in MRI generating fewer false negative studies but a greater number of false positive studies, which can result in unnecessary biopsy $(14,15)$. Additionally, studies have shown that screening with MRI is not costeffective in women with lower to average risk for breast cancer, which is reflected in its omission for these groups in the current American Cancer Society (ACS) recommendations $(16,17)$.

Women with genetic mutations associated with an increased risk for breast cancer, history of previous mantle radiation or those with an estimated lifetime risk greater than $20 \%$, based on risk stratification tools, are classified as high risk for breast cancer (18). For these individuals, several organizations have recommended breast MRI for screening as an adjunct to mammography (19-22). The Claus model is the only validated model which predicts benefit from screening MRI, which mostly takes into account a woman's age and family history (23). Alternative models such as the Tyrer-Cuzick (TC), the Breast and Ovarian Analysis of Disease Incidence and Carrier Estimation Algorithm (BOADICEA) and Gail attempt to be more comprehensive and include both family history, as well as non-familial risk factors $(24,25)$. Due to inherent differences in the data included in these models, there can be great variability in mathematical risk calculation, which can impact screening recommendations. In a previous study, 33 women were evaluated for MRI-based breast screening. Using $20 \%-25 \%$ lifetime risk as a minimum cutoff for MRI, the Claus model identified one eligible patient, while alternative models such as Gail model and the TC model identified nine and 12 eligible patients, respectively (26). The authors did not determine the benefit patients received from enhanced screening, such as an increase in cancer detection.

The Gail and TC models are readily available online risk calculators that account for family history, personal history and modifiable factors in some variation to determine risk. Currently the TC model is used to guide MRI screening eligibility for high-risk patients, whereas the Gail model has been designed to guide use of chemoprevention as determined by the NSABP STAR trial (27). In our study, we compared the TC and Gail Lifetime Risk (LR\%) and their correlation with biopsy proven breast cancer diagnosis subsequent to MRI screening. We also aimed to determine if the largely accepted $20 \%$ lifetime risk is associated with a statistical increase in cancer detection in a cohort of

\section{Materials and Methods}

After receiving IRB approval, we performed a retrospective review of the Virginia Commonwealth University (VCU) Imaging Database from January 2005 to December 2015. We evaluated patients who received screening breast MRI as an adjunct to mammography, based on a variety of reasons including: presence of genetic mutations such as BRCA1/BRCA2; presence of atypia or other high-risk lesions on previous biopsy; LR\% greater than $20 \%$ on TC or other risk models; presence of extensive breast or ovarian family history; or presence of extremely dense breasts on mammography.

The cohort included females, aged 18 to 75 , who underwent screening breast MRI between January 2005 to December 2015 within a VCU Health affiliated hospital. In addition to screening MRI, patients received screening mammography, alternating mammogram and MRI every six months. Subjects who received a diagnostic breast MRI due to a diagnosis of breast cancer were excluded from the study. Additionally, subjects with a prior history of breast cancer, or those with breast cancer diagnosis with a screening method other than MRI, that is ultrasound, were also excluded.

We collected clinical and pathological data for all subjects. Variables associated with an increased risk of breast cancer including race, body mass index (BMI), parity, age at first birth, genetic testing, age of menarche, menopausal status, HRT and family history for breast cancer including first- and second-degree relatives were collected. Using those variables, we calculated the lifetime risk percentage for future development of breast cancer for every subject in our cohort using both the Gail and TC risk calculators. Of note, we did not calculate Gail risk on subjects aged less than 35 years at first presentation, as the model is not validated in women less than age 35 . We chose not to include a Claus model risk score as it is no longer used in clinical practice. We recorded the results of the MRI report as well as patient age at the time of the first MRI used for screening and the age for patients diagnosed with biopsy proven breast cancer using MRI as a method for screening.

Ethics committee approval was obtained from Virginia Commonwealth University Institutional Review Board and the approval was given on May 31, 2018.

\section{Statistical Analysis}

We compared the accuracy of the Gail and TC models as lifetime risk calculators for breast cancer detection by calculating the receiver operating characteristic (ROC) curves for each test separately. The Gail model was considered our control model as it is a well validated standardized risk model used for other purposes but is not considered validated for determining utility of MRI. ROC curves are popular tools summarizing the trade-off between true positive and false positive rates for a predictive model (corresponding to the competing tests in this study) under various probability thresholds. Comparison of the ROC curves via the calculated area under the curve (AUC) corresponding to the Gail and TC models was performed using DeLong's test. Additionally, Fisher's Exact Test was utilized to determine the significance of cancer detection with screening MRI when the TC or Gail LR percentages are greater than $20 \%$. A p-value of $<0.05$ was considered statistically significant for our analyses. All statistics were performed using SAS Software, version 9.4 (Cary, NC., USA). 


\section{Results}

We identified 163 subjects in the VCU breast imaging database eligible for the study based on inclusion criteria. A total of five subjects were diagnosed with biopsy proven breast cancer after undergoing screening with MRI, representing $3.1 \%$ of our patient cohort. The mean age at first screening MRI was 48.2 years and the mean age at cancer diagnosis was 41.4 years (Table 1). The mean lifetime risk of developing breast cancer according to TC version 7 and Gail model was $25.5 \%$ and $16.9 \%$, respectively. Furthermore, $20.2 \%$ of our cohort had undergone a prior breast biopsy with $24.2 \%$ having findings such as atypia, or lobular carcinoma in situ (LCIS). The majority (90.8\%) of subjects had a first degree relative with known breast cancer and $71.8 \%$ were parous with a mean age of parity at 26.6 years. Lastly, 49 patients had undergone prior genetic testing with 19 testing positive for $B R C A 1 / B R C A 2$ or other hereditary unspecified genetic mutations (Table 1).

Logistic ROC analysis results showed that the AUC scores for TC and Gail were 0.6841 and 0.6543 , respectively. There was no significant difference in predictive ability between the two calculators $(\mathrm{p}=0.828)$ (Figure 1).

In order to determine whether utilizing a $20 \%$ lifetime breast cancer risk as an MRI screening cutoff clinically improves cancer detection, the relationship between biopsy proven breast cancer diagnosis with the Gail and TC calculators was explored when the cutoff value was set at $20 \%$. Based on available information from electronic medical records, the Gail model was utilized in 134 of the subjects. (remaining subjects were age $<35$ years and did not qualify for Gail LR calculation). One hundred subjects were determined to have a LR $\leq 20 \%$, with four subjects in this cohort later developing biopsy proven breast cancer (Table 2). Thirty-four subjects were determined to have LR\% greater than $20 \%$, with one subject later being diagnosed with breast cancer. There was no statistically significant difference in the diagnosis of breast cancer between the two Gail groups ( $\mathrm{p}=$ 1.0) (Table 2). There were a total of 163 calculated TC lifetime risk

\section{Table 1. Population demographics}

\begin{tabular}{ll} 
Number of subjects in the study & 163 \\
Mean age first screening MRI & 48.2 \\
Mean age of menarche & 12.5 \\
Mean BMI & 28.9 \\
Percentage of parity & 71.8 \\
Mean age of parity & 26.6 \\
Percentage with a breast biopsy & 20.2 \\
\hline Percentage with atypia/LCIS in biopsy result & 24.2 \\
Percentage of first-degree relatives with breast cancer & 90.8 \\
Average Percentage of TC score & 25.5 \\
Average Percentage of Gail Score & 16.9 \\
Mean age of biopsy confirmed breast CA & 41.4 \\
\hline Number of patients with genetic testing & 49 \\
\hline $\begin{array}{l}\text { Number of patients with genetic mutations known to } \\
\text { predispose to breast cancer (eg. BRCA1, BRCA2) }\end{array}$ & 19 \\
\hline $\begin{array}{l}\text { MRI: Magnetic resonance imaging, BMl: Body mass index, LCIS: Lobular } \\
\text { carcinoma in situ, TC: Tyrer-Cuzick, CA: Cancer }\end{array}$
\end{tabular}

percentages, with 78 corresponding subjects receiving $\leq 20 \%$ and 85 subjects receiving greater than $20 \%$ (Table 3). One subject with LR\% less than or equal to $20 \%$ later developed biopsy proven breast cancer, while four subjects belonging in the high-risk group were diagnosed with malignancy during the study period. There was no statistically significant difference in the diagnosis of breast cancer between the two groups $(\mathrm{p}=0.369)$ (Table 3$)$.

\section{Discussion and Conclusion}

Breast cancer risk calculators can provide valuable information that can be used to guide prevention, screening and chemoprophylaxis strategies in women. The Gail model, while not intended to determine MRI eligibility, has been utilized to guide chemoprophylaxis eligibility in women with a 5 -year breast cancer risk of $1.67 \%$ or higher $(28$, 29). In contrast, the TC, in addition to the Claus and BOADICEA models, has been used to determine MRI eligibility for screening

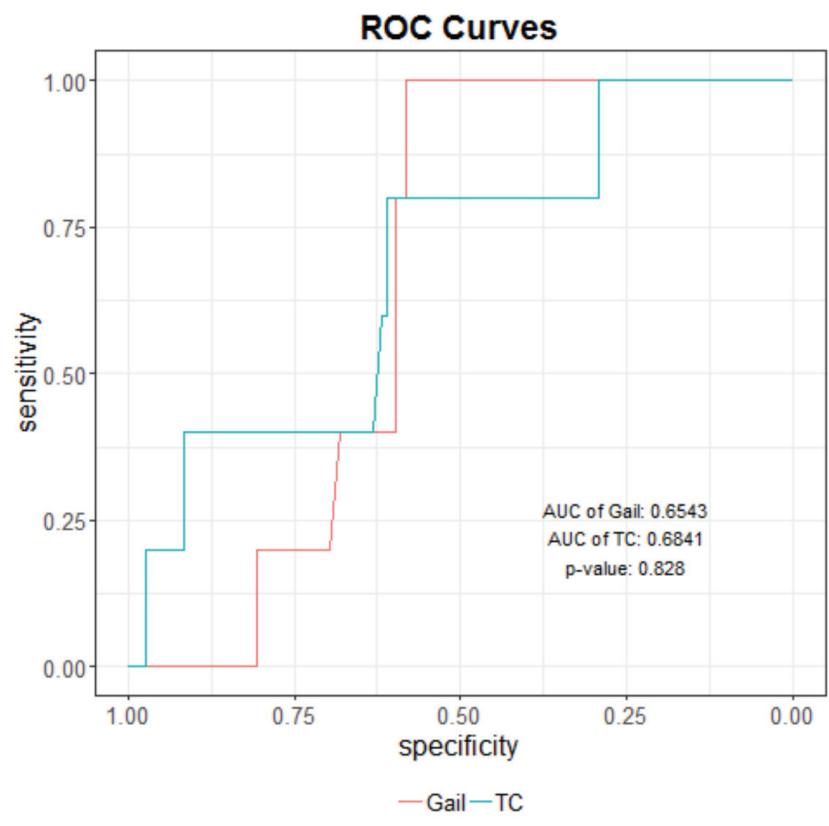

Figure 1. ROC curves of the Gail (red line) and TC (blue line) models when predicting MRI detection of breast cancer

ROC: Receiver operating characteristics curve, TC: Tyrer-Cuzick, MRI: Magnetic resonance imaging, AUC: Area under the curve

Table 2. Subject frequency and percentage of the diagnosis of breast cancer in Gail Risk Score Group [low risk ( $\leq 20 \%)$ vs. high risk (>20\%)]

\begin{tabular}{lccc} 
& \multicolumn{3}{c}{ Breast cancer diagnosis } \\
$\begin{array}{l}\text { Gail group } \\
(\%)\end{array}$ & $\begin{array}{c}\text { No } \\
\text { n (\%) }\end{array}$ & $\begin{array}{c}\text { Yes } \\
\mathbf{n}(\%)\end{array}$ & $\begin{array}{c}\text { Total } \\
\mathbf{n}(\%)\end{array}$ \\
$\mathbf{5 2 0}$ & $96(96.0)$ & $4(4.0)$ & 100 \\
$>\mathbf{2 0}$ & $33(97.1)$ & $1(2.9)$ & 34 \\
Total & 129 & 5 & 134
\end{tabular}

The Fisher's exact test p-value 1.00 indicated that there was no statistically significant difference in diagnose breast cancer between the two Gail groups. 
purposes (30). These risk assessment models, while commonly used in clinical practice, have been shown to have significant variability when identifying different populations of women eligible for screening MRI (31).

While all models have their strengths and weaknesses, the Gail and TC model are the only two that are readily available and free to all users. The Gail model contains fewer factors and can be easily run by patients themselves. However, it is not validated in women less than age 35 years, which limits its usefulness as a risk model for younger patients. TC is a more complex and robust risk model. However, it can be difficult to use and requires a provider to enter data, thus limiting its use outside of the clinic $(24,25)$. Conflicting data exist in the literature regarding the level of accuracy between these two models, with some studies indicating that the TC model is superior in terms of specificity, sensitivity, and positive and negative predictive value $(32,33)$, while others reporting greater AUC and specificity for the Gail model $(34,35)$. Guidelines warn against the use of the Gail model when assessing MRI eligibility for screening purposes due to accounting for limited family history (31). The TC model collects additional data, such as menopausal status, BMI, more extensive family history and the presence of LCIS, which theoretically can increase breast cancer risk prediction (Table 4). Additionally, variables such as mammographic density and genetic and non-genetic factors have been supported to aid in improved cancer risk prediction (36). When accounting for all the additional risk factors that the TC model takes into account, our data suggest that the TC lifetime risk percentage offers no additional accuracy in predicting breast cancer detection by MRI than the Gail model. These findings are supported by a recent study which found that the TC lifetime risk percentage failed to identify approximately $40 \%$ of women who were eligible for changes in their medical management, such as undergoing screening MRI (37) and another large cohort study that reported significant overestimation of breast cancer with the TC model when high risk lesions are found (38).

Breast MRI has been recommended as an adjunct to mammography in women classified as high-risk for development of breast cancer. The recommendations stem from a consensus panel which determined that a Claus LR\% equal or greater than $20 \%$ is associated with increased cancer detection. The Claus model takes into account hereditary risk factors but fails to include non-

Table 3. Subject frequency and percentage of the diagnosis of breast cancer in TC risk score [low risk ( $\leq 20 \%)$ vs. high risk $(>20 \%)]$

\begin{tabular}{lccc}
\multicolumn{4}{c}{ Breast cancer diagnosis } \\
$\begin{array}{l}\text { TC group } \\
(\%)\end{array}$ & No & Yes & Total \\
$\mathbf{n}(\%)$ & $\mathbf{n}(\%)$ & $\mathbf{n}(\%)$ \\
$\mathbf{5 2 0}$ & $77(98.7)$ & $1(1.3)$ & 78 \\
$>\mathbf{2 0}$ & $80(95.2)$ & $4(4.8)$ & 85 \\
Total & 158 & 5 & 163
\end{tabular}

The Fisher's exact test p-value 0.3689 indicated that there was no statistically significant difference in diagnose breast cancer between the two TC groups

TC: Tyrer-Cuzick hereditary risk factors that have been found to impact the lifetime risk of breast cancer in a woman. Since the TC and Gail models additionally account for non-hereditary risk factors and are widely available online, they are routinely used for risk stratification of MRI eligibility and chemoprophylaxis management, respectively. The $20 \%$ cutoff associated with increased cancer detection remains a criterion for classifying a woman as high-risk for breast cancer development, irrespective of the limitations of the Claus model. The TC and Gail models vary from the Claus model, as demonstrated in previous studies, with the TC and Gail models estimating a far higher lifetime risk than Claus (26). In fact, a more recent study found significant differences in the number of women that were eligible for MRI screening identified by the risk assessment models utilized in the study (TC, Claus, BRCAPRO) (31). In our study, we demonstrated no statistically significant correlation between the Gail or TC models when utilizing MRI as a screening modality with $20 \%$ lifetime risk cutoff to classify patients as high-risk. While the TC model is a rich source of information and risk stratification, this information calls into question the common practice of using $20 \%$ lifetime risk as cutoff for yearly MRI screening when the TC model is used to determine risk. Our data, along with others, suggest the $20 \%$ LR, as determined by testing the Claus model, may be too low when using a more sensitive model such as TC.

The results of our study should be interpreted in the context of its limitations. A major limitation of our study was the limited number of subjects who underwent screening MRI at our center and the low number of patients that were diagnosed with biopsy proven breast cancer after undergoing screening with breast MRI. With only five subjects, or $3.1 \%$ of our high-risk patient population, diagnosed with

Table 4. Variable used in the Claus, Gail and Tyrer-Cuzick models

\begin{tabular}{|c|c|c|c|}
\hline Variables & Gail & Claus & Tyrer-Cuzick \\
\hline \multicolumn{4}{|l|}{ Personal information } \\
\hline Age & Yes & Yes & Yes \\
\hline Body mass index & No & No & Yes \\
\hline \multicolumn{4}{|l|}{ Hormonal factors } \\
\hline Menarche & Yes & No & Yes \\
\hline First live birth & Yes & No & Yes \\
\hline Menopause & No & No & Yes \\
\hline \multicolumn{4}{|l|}{ Personal breast disease } \\
\hline Breast biopsies & Yes & No & Yes \\
\hline Atypical hyperplasia & Yes & No & Yes \\
\hline LCIS & No & No & Yes \\
\hline \multicolumn{4}{|l|}{ Family history } \\
\hline First degree relatives & Yes & Yes & Yes \\
\hline Second degree relatives & No & Yes & Yes \\
\hline Age of onset of cancer & No & Yes & Yes \\
\hline Bilateral breast cancer & No & No & Yes \\
\hline Ovarian cancer & No & No & Yes \\
\hline Male breast cancer & No & No & No \\
\hline
\end{tabular}


breast cancer during the study period, it is possible that our lack of predictive value is due to a low event rate rather than lack of predictive value of either calculator. This study serves only as a pilot study to guide larger trials. A larger prospective clinical trial would be necessary to determine at what percentage lifetime risk we should recommend patients undergo MRI screening when using a more sensitive model such as TC.

In conclusion, the TC model is a risk stratification tool that is currently used to guide breast cancer screening recommendations, while the Gail model has mainly been utilized to guide chemoprophylaxis management in women with increased risk for development of breast cancer. Neither have been validated as a predictive model for utility of MRI screening in a large study. In our study, the TC model did not appear superior to the Gail model when predicting the benefit of breast MRI screening. Additionally, the current $20 \%$ cutoff that classifies a woman as high-risk for future development of breast cancer, which was originally determined based on calculations derived from the Claus model, was not found to be statistically significant between the Gail or TC LR calculators and a diagnosis of breast cancer. These findings suggest that we should use the $20 \%$ LR cutoff using the TC model with caution when making MRI recommendations. A larger, multicenter trial, with a higher event rate of cancer diagnoses would be necessary to determine a more appropriate cutoff value for initiating MRI screening using this widely available risk calculator.

Acknowledgements: Services and products in support of this research project were generated by the VCU Massey Cancer Center Biostatistics Shared Resources, supported, in part, with funding from the NIH-NCI Cancer Center Support Grant P30CA016059.

Ethics Committee Approval: Ethics committee approval was obtained from Virginia Commonwealth University Institutional Review Board and the approval was given on May 31, 2018.

Informed Consent: It was obtained.

Peer-review: Externally peer-reviewed.

\section{Authorship Contributions}

Conception: A.S., X.D., D.B., K.P.M.; Design: A.S., X.D., D.B., K.P.M.; Supervision: A.S., X.D., D.B., K.P.M.; Materials: A.S., X.D., D.B., K.P.M.; Analysis and/or Interpretation: A.S., X.D., D.B., K.P.M.; Writing: A.S., X.D., D.B., K.P.M.

Conflict of Interest: No conflict of interest declared by the authors.

Financial Disclosure: This research did not receive any specific grant from funding agencies in the public, commercial, or not-for-profit sectors.

\section{References}

1. Alkabban FM, Ferguson T. Cancer, Breast. In: StatPearls. Treasure Island (FL): StatPearls Publishing; 2020. [Crossref]

2. Howlader N, Noone AM, Krapcho M, et. al. SEER Cancer Statistics Review, 1975-2009. National Cancer Institute. September 7, 2012. [Crossref]

3. Ataollahi MR, Sharifi J, Paknahad MR, Paknahad A. Breast cancer and associated factors: a review. J Med Life. 2015; 8(Spec Iss 4): 6-11. (PMID: 28316699) [Crossref]

4. Engmann NJ, Golmakani MK, Miglioretti DL, Sprague BL, Kerlikowske K. Population-Attributable Risk Proportion of Clinical Risk Factors for Breast Cancer. JAMA Oncol. 2017 Sep 1; 3: 1228-1236. (PMID: 28152151)

5. Doren A, Vecchiola A, Aguirre B, Villaseca P. Gynecologicalendocrinological aspects in women carriers of BRCA1/2 gene mutations. Climacteric. 2018; 21: 529-535. (PMID: 30295091) [Crossref]

6. Punglia RS, Morrow M, Winer EP, Harris JR. Local therapy and survival in breast cancer. N Engl J Med. 2007; 356: 2399-2405. (PMID: 17554121) [Crossref]

7. PDQ Screening and Prevention Editorial Board. Breast Cancer Screening (PDQ ${ }^{\circ}$ ): Health Professional Version. 2019 Dec 18. In: PDQ Cancer Information Summaries [Internet]. Bethesda (MD): National Cancer Institute (US); 2002. (PMID: 26389344) [Crossref]

8. Center for Disease Control and Prevention. Breast Cancer Screening Guidelines for Women. 2017. Available at: https://www.cdc.gov/cancer/ breast/basic_info/screening.htm (Accessed March 9, 2018). [Crossref]

9. Mann RM, Kuhl CK, Moy L. Contrast-enhanced MRI for breast cancer screening. J Magn Reson Imaging. 2019; 50: 377-390. (PMID: 30659696) [Crossref]

10. Kuhl C, Weigel S, Schrading S, Arand B, Bieling H, König R, et al. Prospective multicenter cohort study to refine management recommendations for women at elevated familial risk of breast cancer: The EVA trial. J Clin Oncol. 2010; 28: 1450-1457. (PMID: 20177029) [Crossref]

11. Sardanelli F, Podo F, Santoro F, Manoukian S, Bergonzi S, Trecate G, et al. Multicenter surveillance of women at high genetic breast cancer risk using mammography, ultrasonography, and contrast-enhanced magnetic resonance imaging (the High Breast Cancer Risk Italian 1 Study): Final results. Invest Radiol. 2011; 46: 94-105. (PMID: 21139507) [Crossref]

12. Leibman AJ, Kruse B. Breast cancer: mammographic and sonographic findings after augmentation mammoplasty. Radiology. 1990; 174: 195198. (PMID: 2152981) [Crossref]

13. Jackson VP, Hendrick RE, Feig SA, Kopans DB. Imaging of the radiographically dense breast. Radiology. 1993; 188: 297-301. (PMID: 8327668) [Crossref]

14. Berg WA, Gutierrez L, NessAiver MS, Carter WB, Bhargavan M, Lewis RS, et al. Diagnostic accuracy of mammography, clinical examination, US, and MR imaging in preoperative assessment of breast cancer. Radiology. 2004; 233: 830-849. (PMID: 15486214) [Crossref]

15. Boetes C, Mus RD, Holland R, Barentsz JO, Strijk SP, Wobbes T, et al. Breast tumors: comparative accuracy of MR imaging relative to mammography and US for demonstrating extent. Radiology. 1995; 197: 743-747. (PMID: 7480749) [Crossref]

16. Taneja C, Edelsberg J, Weycker D, Guo A, Oster G, Weinreb J. Cost effectiveness of breast cancer screening with contrast-enhanced MRI in high-risk women. J Am Coll Radiol. 2009; 6: 171-179. (PMID: 19248993) [Crossref]

17. Moore SG, Shenoy PJ, Fanucchi L, Tumeh JW, Flowers CR. Costeffectiveness of MRI compared to mammography for breast cancer screening in a high risk population. JAMA. 2015; 314: 1599-1614. (PMID: 19144138) [Crossref]

18. Saslow D, Bootes C, Burke W, Harms S, Leach MO, Lehman CD, et al; American Cancer Society Breast Cancer Advisory Group. American Cancer Society guidelines for breast screening with MRI as an adjunct to mammography. CA Cancer J Clin 2007; 57: 75-89. Erratum in: CA Cancer J Clin 2007; 57: 185. (PMID: 17392385) [Crossref]

19. Oeffinger KC, Fontham ET, Etzioni R, Herzig A, Michaelson JS, Shih YC, et al. Breast Cancer Screening for Women at Average Risk: 2015 Guideline Update From the American Cancer Society. JAMA. 2015;314(15):1599-1614. (PMID: 26501536) [Crossref]

20. Brawley O, Byers T, Chen A, Pignone M, Ransohoff D, Schenk M, et al. New American Cancer Society process for creating trustworthy cancer 
screening guidelines. JAMA 2011; 306: 2495-2459. (PMID: 22166609) [Crossref]

21. Havrilesky L, Gierisch JM, Moorman P, Havrilesky LJ, Grimm LJ, Ghate S, et al. Systematic Review of Cancer Screening Literature for Updating American Cancer Society Breast Cancer. JAMA 2015; 314: 1615-1634. (PMID: 26501537) [Crossref]

22. Kuhl C, Weigel S, Schrading S, Arand B, Bieling H, König R, et al. Prospective multicenter cohort study to refine management recommendations for women at elevated familial risk of breast cancer: the EVA trial. J Clin Oncol 2010; 28: 1450-1457. (PMID: 20177029) [Crossref]

23. Tyrer J, Duffy SW, Cuzick J. A breast cancer prediction model incorporating familial and personal risk factors. Stat Med 2004; 23: 1111 1130. (PMID: 15057881) [Crossref]

24. Gail MH, Brinton LA, Byar DP, Corle DK, Green SB, Schairer C, et al. Projecting individualized probabilities of developing breast cancer for white females who are being examined annually. J Natl Cancer Inst 1989; 81: 1879-1886. (PMID: 2593165) [Crossref]

25. Hollingsworth AB, Stough RG. An alternative approach to selecting patients for high-risk screening with breast MRI. Breast J 2014; 20: 192 197. (PMID: 24387050) [Crossref]

26. Vogel VG. The NSABP Study of Tamoxifen and Raloxifene (STAR) trial Expert Rev Anticancer Ther 2009; 9: 51-60. Erratum in: Expert Rev Anticancer Ther 2009; 9: 388. (PMID: 19105706) [Crossref]

27. Gail MH, Brinton LA, Byar DP, Corle DK, Green SB, Schairer C, et al. Projecting individualized probabilities of developing breast cancer for white females who are being examined annually. J Natl Cancer Inst 1989; 81: 1879-1886. (PMID: 2593165) [Crossref]

28. Costantino JP, Gail MH, Pee D, Anderson S, Redmond CK, Benichou $\mathrm{J}$, et al. Validation studies for models projecting the risk of invasive and total breast cancer incidence. J Natl Cancer Inst 1999; 91: 1541-1548. (PMID: 10491430) [Crossref]

29. Schenberg T, Mitchell G, Taylor D, Saunders C. MRI screening for breast cancer in women at high risk; is the Australian breast MRI screening access program addressing the needs of women at high risk of breast cancer?. J Med Radiat Sci 2015; 62: 212-225. (PMID: 26451244) [Crossref]
30. Ozanne EM, Drohan B, Bosinoff P, Semine A, Jellinek M, Cronin C, et al. Which risk model to use? clinical implications of the ACS MRI screening guidelines. Cancer Epidemiol Biomarkers Prev. 2013; 22: 146149. (PMID: 23093547) [Crossref]

31. Vianna FSL, Giacomazzi J, Oliveira Netto CB, et al. Performance of the Gail and Tyrer-Cuzick breast cancer risk assessment models in women screened in a primary care setting with the FHS-7 questionnaire. Genet Mol Biol 2019; 42(Suppl 1): 232-237. (PMID: 31170278) [Crossref]

32. Zhang L, Jie Z, Xu S, Zhang L, Guo X. Use of Receiver Operating Characteristic (ROC) Curve Analysis for Tyrer-Cuzick and Gail in Breast Cancer Screening in Jiangxi Province, China. Med Sci Monit. 2018; 24: 5528-5532. (PMID: 30089770) [Crossref]

33. McCarthy AM, Guan Z, Welch M, Griffin ME, Sippo DA, Deng Z, et al. Performance of Breast Cancer Risk-Assessment Models in a Large Mammography Cohort. J Natl Cancer Inst 2020; 112: 489-497. (PMID: 31556450) [Crossref]

34. Stevanato KP, Pedroso RB, Iora P, Dos Santos L, Pelloso FC, de Melo WA, et al. Comparative Analysis between the Gail, Tyrer-Cuzick and BRCAPRO Models for Breast Cancer Screening in Brazilian Population. Asian Pac J Cancer Prev 2019; 20: 3407-3413. (PMID: 31759366) [Crossref]

35. Brentnall AR, Cuzick J, Buist DSM, Bowles EJA. Long-term Accuracy of Breast Cancer Risk Assessment Combining Classic Risk Factors and Breast Density. JAMA Oncol 2018; 4: e180174. (PMID: 29621362) [Crossref]

36. Gorringe HM, Rosenthal E, Morris B, Manley S. Genetic testing contributes significantly to improved identification of women eligible for increased breast cancer screening compared to the Tyrer-Cuzick risk model [abstract]. AACR; Cancer Res 2019; 79. doi: 10.1158/1538-7445. SABCS18-P4-03-04 [Crossref]

37. Boughey JC, Hartmann LC, Anderson SS, Degnim AC, Vierkant RA, Reynolds CA, et al. Evaluation of the Tyrer-Cuzick (International Breast Cancer Intervention Study) model for breast cancer risk prediction in women with atypical hyperplasia. J Clin Oncol 2010; 28: 3591-3596. (PMID: 20606088) [Crossref] 